\title{
Graphene Oxide Reinforced Polylactic Acid/Polyurethane Antibacterial Composites
}

\author{
Xiaoli An, ${ }^{1,2,3}$ Haibin $\mathrm{Ma}^{4}{ }^{4}$ Bin Liu, ${ }^{3}$ and Jizeng Wang ${ }^{1,2}$ \\ ${ }^{1}$ Key Laboratory of Mechanics on Environment and Disaster in Western China, The Ministry of Education of China, \\ Lanzhou 730000, China \\ ${ }^{2}$ Institute of Solid Mechanics, School of Civil Engineering and Mechanics, Lanzhou University, Lanzhou 730000, China \\ ${ }^{3}$ School of Stomatology, Lanzhou University, Lanzhou 730000, China \\ ${ }^{4}$ Department of Stomatology, Traditional Chinese Medicine Hospital of Gansu Province, Lanzhou 730050, China
}

Correspondence should be addressed to Bin Liu; liubkq@lzu.edu.cn and Jizeng Wang; jzwang@lzu.edu.cn

Received 13 June 2013; Accepted 12 August 2013

Academic Editor: Xingbin Yan

Copyright (c) 2013 Xiaoli An et al. This is an open access article distributed under the Creative Commons Attribution License, which permits unrestricted use, distribution, and reproduction in any medium, provided the original work is properly cited.

Nanocomposites from PLA/PU containing small concentrations of graphene oxide (GO) were prepared by simple liquid-phase mixing followed by casting. The as-prepared ternary PLA/PU/GO composite films exhibited good antibacterial activity against the gram-positive Staphylococcus aureus and the gram-negative Escherichia coli, due to the excellent antibacterial property of GO sheets with high specific surface area. The addition of GO inhibited the attachment and proliferation of microbes on the film surfaces, resulting in that the PLA/PU/GO composite films show remarkably improved antibacterial activity compared with PLA/PU composite film. The inhibition efficiency is proportional to the amount of GO. Furthermore, PLA/PU/GO composite fibrous paper was fabricated using electrospinning and exhibited good biocompatibility. The addition of GO does not destroy normal cell's proliferation and differentiation. PLA/PU/GO composites with good antibacterial activity and biocompatibility make it attractive for the environmental and clinical applications and also provide a candidate for future application of tissue engineering.

\section{Introduction}

Tissue engineering is a field of research in biomedical engineering that applies the principles and methods of engineering and life sciences toward the fundamental understanding of structure/function relationships in normal and pathological mammalian tissues and the development of biological substitutes to restore, maintain, or improve functions [1]. This technique involves processes such as seeding cells onto a scaffold, creating artificial organs and tissues, and triggering or modulating new tissue formation. Currently, synthetic biomaterials (bioceramics and biopolymers) have been widely applied to the tissue engineering fields [26]. Also, antimicrobial materials have attracted considerable attention because bacterial infection is a major medical complication associated with the use of implanted medical devices [7-9]. Bacterial infection may result from tissue destruction, premature device failure, and the spread of the infection from other areas $[8,10]$. Therefore, much effort has been expended to improve antibacterial activity of synthetic biomaterials [11-13].

As thermoplastic synthetic polymers with desirable mechanical property profile, biocompatibility and biodegradability, polylactic acid (PLA) and polyurethane (PU) are two of the most promising biopolymers for tissue engineering territory, such as bone scaffolds, vascular grafts, and heart valves $[14,15]$. Moreover, PU exhibits a certain degree of antibacterial efficacy $[16,17]$. Compared with scaffolds fabricated from PLA or PU alone [18], it has been known that the composite PLA/PU scaffold exhibits advantageous morphology, mechanics, cell adhesion, and growth-supporting properties. However, dissatisfactory antibacterial activity coming from PU phase in PLA/PU composite limits its practical application in tissue engineering fields.

Researchers had excitedly turned to the material to discover graphene's potential applications, since it was first isolated with the help of Scotch tape in 2004 [19]. Graphene and graphene oxide (GO), with extraordinary mechanical 
properties (high Young's modulus and hardness and excellent flexibility) [20], high specific surface area, and low cost, have been considered to be effective reinforcements for high-performance composites [21-24]. On the other hand, GO contains a number of oxygen-containing groups, which makes it relatively easy to be dispersed into some polar solvents and form intercalated composites with polar molecules through the strong interaction [25-30]. Recently, graphene and GO have been proved to exhibit excellent biocompatibility and high antibacterial activity [25, 30-34]. These have motivated us to explore the possibility of GO as an antibacterial reinforcement in biomaterials and study the antiseptic properties and cytotoxicity of GO-containing composites.

In this study, PLA/PU/GO composite films were prepared by introducing GO nanosheets into PLA and PU phases through liquid-phase mixing and casting. The as-synthesized composites are characterized by using transmission electron morphology, X-ray diffraction, and field emission scanning electron microscope. Moreover, we investigate the antibacterial activity and biocompatibility of PLA/PU/GO. It showed remarkably improved antibacterial activity compared with PLA/PU composite. The addition of small amounts of GO prevented the attachment and proliferation of microbes on PLA/PU/GO surfaces, including the gram-positive Staphylococcus aureus (S. aureus) and the gram-negative Escherichia coli (E. coli), as causative organisms of many device-related infections. PLA/PU/GO composites have excellent antibacterial activity and biocompatibility, suggesting their potential application in tissue engineering.

\section{Experimental}

2.1. Synthesis of PLA/PU/GO Composite Films. Polyurethane (PU) was synthesized in our laboratory according to previous study $[35,36]$. GO nanosheets were prepared according to the method described by Hummer with a modification [37, 38]. In brief, powdery GO was dispersed in a $50 \mathrm{~mL}$ of DMF and dichloromethane (volume ratio $=2 / 3$ ) solution by ultrasonification to form a uniform suspension. After that, 12 wt.\% PLA $(\mathrm{Mw}=26,000)$ and 3 wt.\% PU (mass ratio $=4 / 1$ ) were dissolved into the suspension with the aid of ultrasonification and stirring. Subsequently, PLA/PU/GO composite film was obtained by casting the suspension onto a clean silicon wafer, evaporating organic solvents, drying at $60^{\circ} \mathrm{C}$ in vacuum for $24 \mathrm{~h}$, and peeling from the Si wafer. In our system, the PLA/PU/GO composite films with two different $\mathrm{GO}$ contents (the weight ratios between GO and polymers were $3: 100$ and $5: 100)$ were prepared, which were denoted as PLA/PU/GO (3\%) and PLA/PU/GO (5\%), respectively. In comparison, PLA/PU film was prepared under the same conditions.

2.2. Synthesis of PLA/PU/GO Composite Nanofibers. PLA/ $\mathrm{PU} / \mathrm{GO}$ nanocomposite fibrous membrane was prepared by electrospinning. In brief, the above PLA/PU/GO (5\%) suspension was used as the electrospun precursor. In a typical electrospinning process, the precursor suspension was loaded into a plastic syringe equipped with a 23-gauge stainless steel needle. A high voltage of $16 \mathrm{kV}$ was supplied by a directcurrent power supply and the feeding rate for the precursor suspension was adjusted to a constant rate of $0.3 \mathrm{~mL} / \mathrm{h}$ using a syringe pump. A piece of aluminum foil was placed $15 \mathrm{~cm}$ below the tip of the needle to collect the as-spun nanofibers. Finally, the PLA/PU/GO fibrous paper was obtained by removing residual solvents at room temperature and peeling from the foil. Also, PLA/PU nanofibrous paper was prepared under the same conditions.

2.3. Characterization. Transmission electron microscopy (TEM, JEOL, JEM-2010) was employed to investigate the morphology of as-prepared GO, using an accelerating voltage of $200 \mathrm{kV}$. XPS measurement of the GO was performed on a Perkin-Elmer PHI-5702 multi-functional X-ray photoelectron spectroscope (Physical Electronics, USA), using Al$\mathrm{K} \alpha$ radiation (photon energy: $1476.6 \mathrm{eV}$ ) as the excitation source and the binding energy of $\mathrm{Au}\left(\mathrm{Au} 4 \mathrm{f}_{7 / 2}: 84.00 \mathrm{eV}\right)$ as the reference. X-ray diffraction (XRD) patterns were carried out by an X-ray diffraction using $\mathrm{CuK \alpha}$ radiation (XRD, Rigaku, D/Max-2400), to investigate the microstructures of the PLA/PU/GO composite samples. Field emission scanning electron microscope (FESEM, JEOL, JSM 6701F) was employed to investigate the cross section of the casted films and the morphology of as-electrospun nanofibers.

2.4. Antibacterial Activity Test. The antibacterial activity of PLA/PU and PLA/PU/GO nanocomposites against $E$. coli ATCC 25922 and S. aureus ATCC 6538 was measured by using plastics-measurement of antibacterial activity on plastics surfaces (ISO 22196, International Standard $22196: 2007(\mathrm{E})$ ). Before the test, the film samples (PLA/PU, $\mathrm{PLA} / \mathrm{PU} / \mathrm{GO}(3 \%)$ and PLA/PU/GO (5\%)) were cut into $40 \mathrm{~mm} \times 40 \mathrm{~mm}$ and washed repeatedly with sterile water, then dried in the air. At the same time, polyethylene films were prepared in $40 \mathrm{~mm} \times 40 \mathrm{~mm}$, sterilized in $75 \%$ ethanol, and then washed with sterile water. $1.5 \times 10^{5} \mathrm{CFU} / \mathrm{mL}$ bacterial suspension was seeded on each film sample and covered with polyethylene film so that the bacterial suspension can spread to the edges. Make sure that bacterial suspension does not leak beyond the edges of the film. $40 \mathrm{~mL}$ of phosphate-buffered saline (PBS) was used to wash the sample and polyethylene films in order to get the present bacteria followed by gentle shaking for $1 \mathrm{~h}$, then diluted to $10-$ 100 -fold with a gradient method, and $1 \mathrm{~mL}$ of each dilution was placed into separate sterile Petri dishes. After that, $15 \mathrm{~mL}$ of plate count agar was poured into each Petri dish to disperse the bacteria by swirling gently. All plating shall be performed in duplicate. The Petri dishes were inverted and incubated at $37^{\circ} \mathrm{C}$ for $24 \mathrm{~h}$. After incubation, the number of colonies in the Petri dishes was counted. For each dilution series, record the number of colonies recovered to two significant figures, as well as the dilution factor for the plates used for counting.

$S$. aureus and $E$. coli were seeded on glass plate and PLA/PU/GO (5\%) film and incubated for $24 \mathrm{~h}$. The glass plate and PLA/PU/GO (5\%) film seeded with bacteria were rinsed twice with PBS and fixed in 3\% glutaraldehyde for $4 \mathrm{~h}$, then 


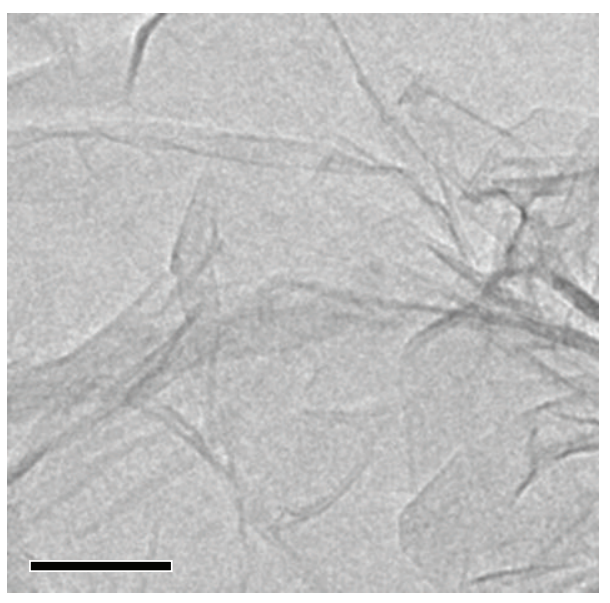

(a)

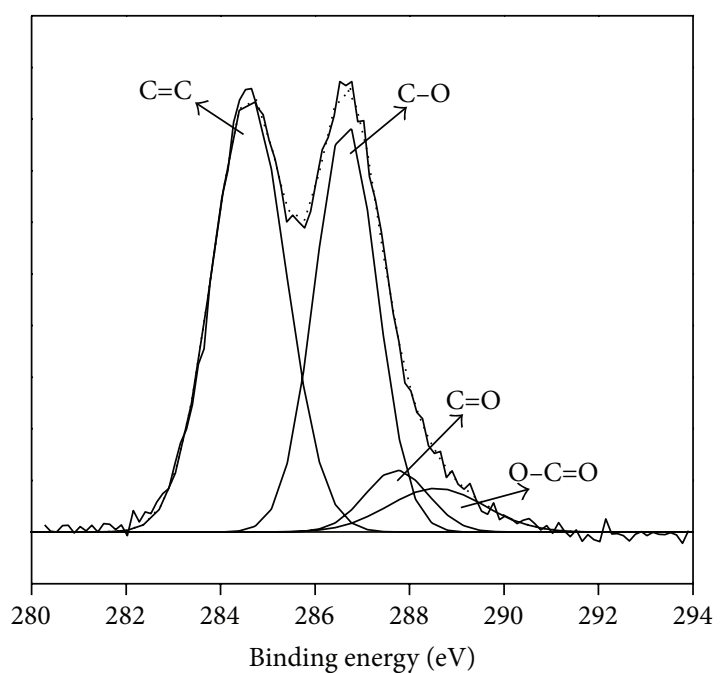

(b)

FIGURE 1: Characterization of GO sheets: (a) TEM image and (b) C1s XPS spectrum. The scale is $100 \mathrm{~nm}$.

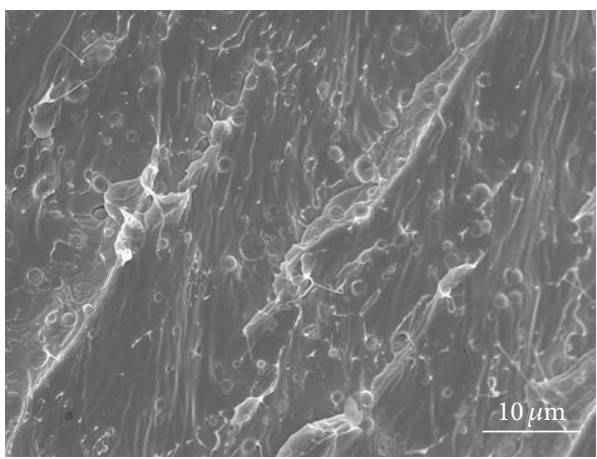

(a)

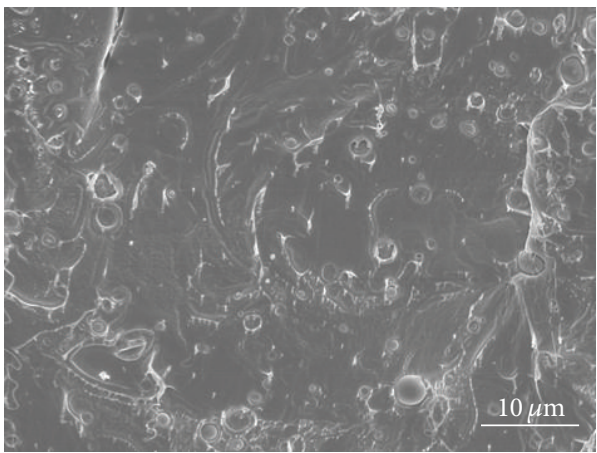

(c)

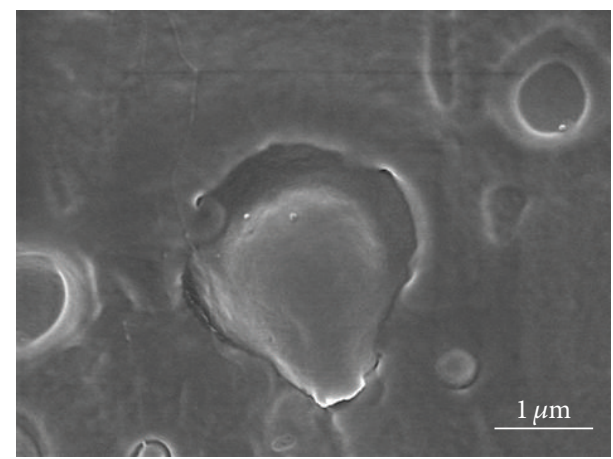

(b)

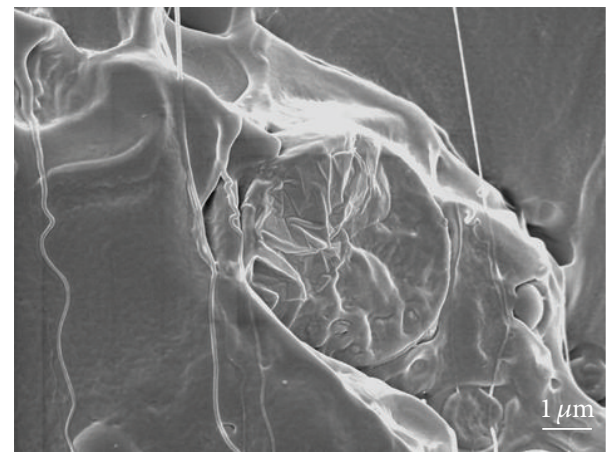

(d)

FIgURE 2: Cross section SEM images of PLA/PU film ((a) and (b)) and PLA/PU/GO (5\%) film ((c) and (d)) with different magnifications.

rinsed in $0.1 \mathrm{~mL}$ of PBS. Thereafter, they were dehydrated with upgrading concentrations of ethanol $(30 \%, 50 \%, 60 \%$, $70 \%, 80 \%, 90 \%, 95 \%$, and $100 \%$ ) twice for 15 min each. After final washing with $100 \%$ ethanol, the samples were kept in fume hood. Finally, the glass plate and PLA/PU/GO (5\%) film were sputter-coated with gold and observed under SEM to examine the morphology of E. coli and S. aureus.
2.5. Biocompatibility Test. To study the biocompatibility of the fibrous samples, MC3T3-E1 cells were used in our system, which are commonly used to assess cytotoxicity of materials. MC3T3-E1 cells were maintained at RPMI-1640 medium with $10 \%$ fetal bovine serum and $1 \%$ antibiotic/antimycotic solution in a $5 \% \mathrm{CO}_{2}$ incubator at $37^{\circ} \mathrm{C}$, refreshed every 3 days with Trypsin-EDTA solution, and then resuspended 


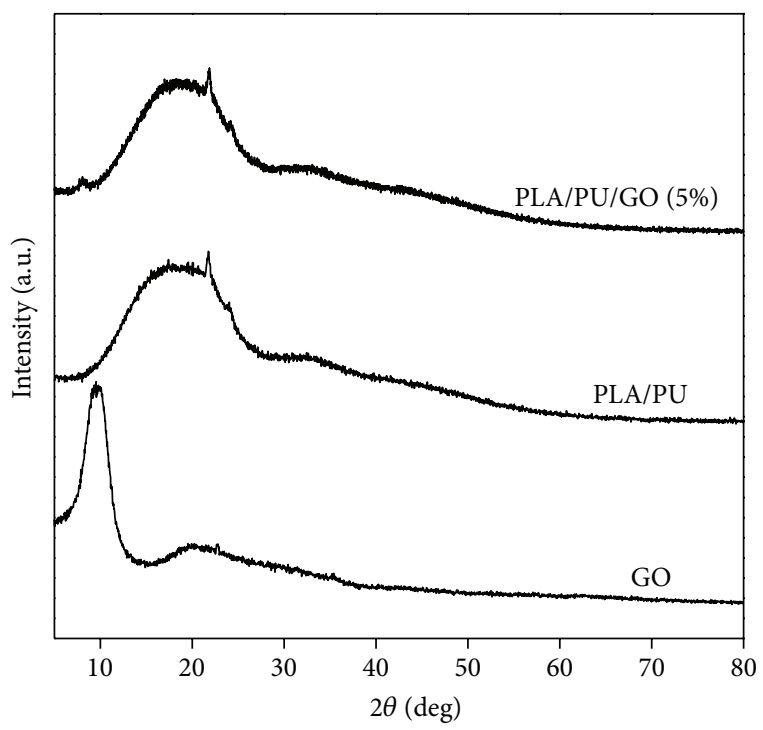

FIgUre 3: XRD patterns of GO, PLA/PU, and PLA/PU/GO (5\%).

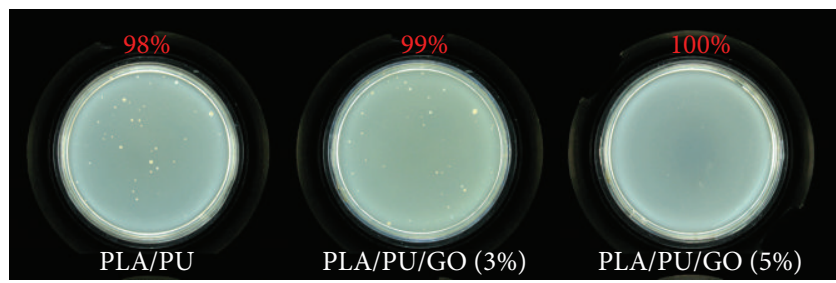

(a)

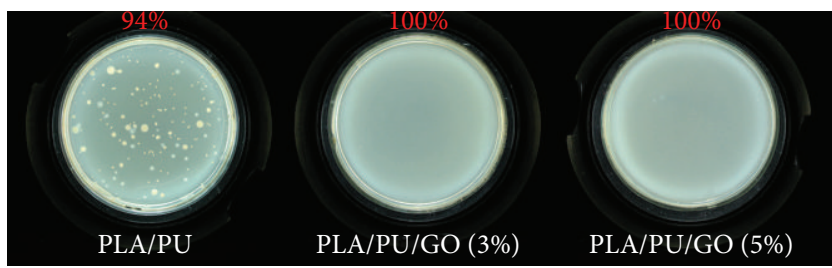

(b)

Figure 4: Photographs of (a) S. aureus and (b) E. coli grown on PLA/PU, PLA/PU/GO (3\%), and PLA/PU/GO (5\%) for $24 \mathrm{~h}$, respectively.

in a fresh culture medium. The PLA/PU and PLA/PU/GO (5\%) fibrous papers were cut into pieces $(1 \mathrm{~cm} \times 1 \mathrm{~cm})$ as scaffolds, soaked in $75 \%$ ethanol for $1 \mathrm{~h}$ to be sterilized, and then exchanged with PBS for three times ( 30 min each). The fibrous scaffolds were then washed with RPMI-1640 containing 10\% FBS for two times ( $2 \mathrm{~h}$ each). $2 \times 10^{5}$ cell suspension was seeded on each scaffold. The cell-scaffold constructs were cultured in RPMI-1640 supplemented with $10 \%$ FBS, $1 \%$ antibiotic/antimycotic solution for up to $48 \mathrm{~h}$. Finally, the cells were stained with acridine orange (AO), which was cleaved to yield a green fluorescent product by metabolically active cells. The density of the cells that adhered on each scaffold was observed at 100-fold magnification with a fluorescence microscopy (Olympus BX51).

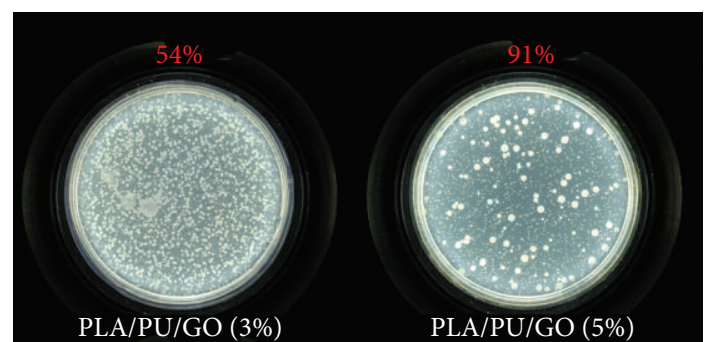

(a)

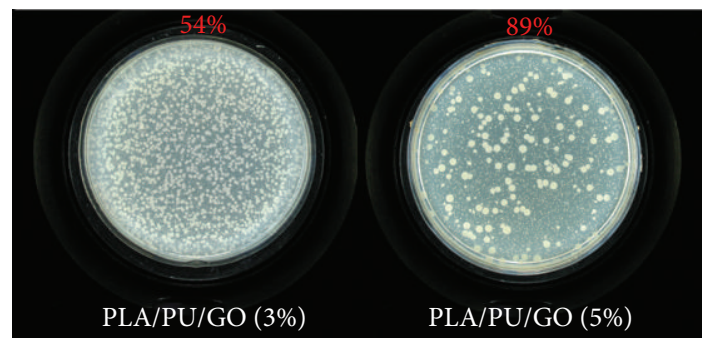

(b)

Figure 5: Photographs of (a) S. aureus and (b) E. coli grown on PLA/PU/GO (3\%) and PLA/PU/GO (5\%) for $4 \mathrm{~h}$, respectively.

\section{Results and Discussions}

As shown in Figure 1(a), as-prepared GO sheets are nearly transparent under electron irradiation, indicating that the GO sheets are quite thin. As shown in Figure 1(b), the C1s XPS spectrum of GO indicates the presence of four components: the $\mathrm{C}$ in $\mathrm{C}=\mathrm{C}$ bonds $(284.5 \mathrm{eV})$, the $\mathrm{C}$ in $\mathrm{C}-\mathrm{O}$ bonds $(286.6 \mathrm{eV})$, the $\mathrm{C}$ in $\mathrm{C}=\mathrm{O}$ bonds $(287.7 \mathrm{eV})$, and the $\mathrm{C}$ in $\mathrm{O}-\mathrm{C}=\mathrm{O}$ bonds $(288.7 \mathrm{eV})$. It indicates the considerable degree of the oxidation existing in GO material $[39,40]$. GO sheets are a chemically modified graphene with suspended hydroxyl, epoxyl, and carboxyl functional groups [41], which result in the hydrophilic nature of GO.

As we know, fully exfoliated GO nanosheets can be dispersed in a polar organic solvent N,N-dimethylformamide (NMF) without any assistance of chemical treatment [42]. In our system, PLA/PU/GO composite films were prepared by a simple liquid-phase mixing followed by casting. Figure 2 shows the SEM images of the fracture surfaces of the PLA/PU and PLA/PU/GO (5\%) films.

As low-magnification images show (Figures 2(a) and 2(c)), two fracture surfaces exhibit the same morphology: the PU particles are randomly distributed within the PLA polymer matrix without obvious agglomeration. However, high-magnification images (Figures 2(b) and 2(d)) show that their fracture surfaces are totally different. For the fracture of the PLA/PU film, the surface of the individual PU particle is relatively smooth. In comparison with the fracture of the PLA/PU/GO (0.5\%) film, the surface of the individual PU particle is wrapped by layer-stacked GO sheets. Our fabrication process is presented to explain the combination of GO sheets and PU particles in PLA polymer matrix. As we know, PU molecule chains contain plenty of $\mathrm{N}-\mathrm{H}$ groups and GO sheets contain many oxygen-containing groups. After 


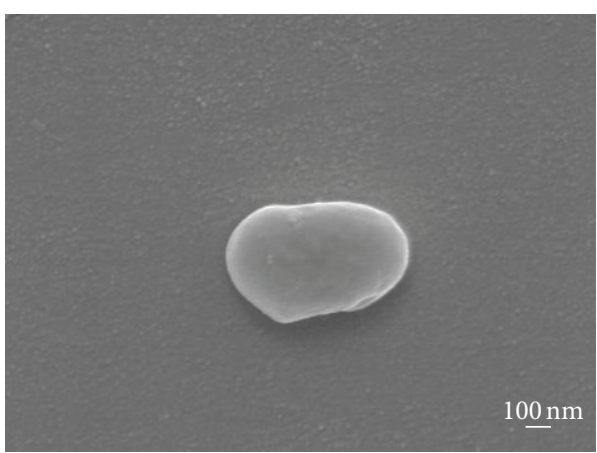

(a)

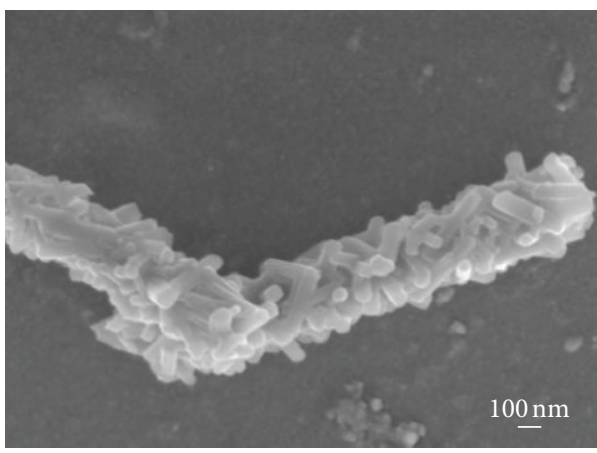

(c)

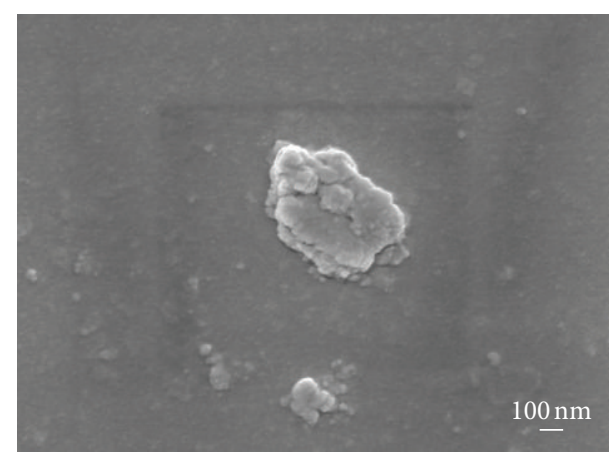

(b)

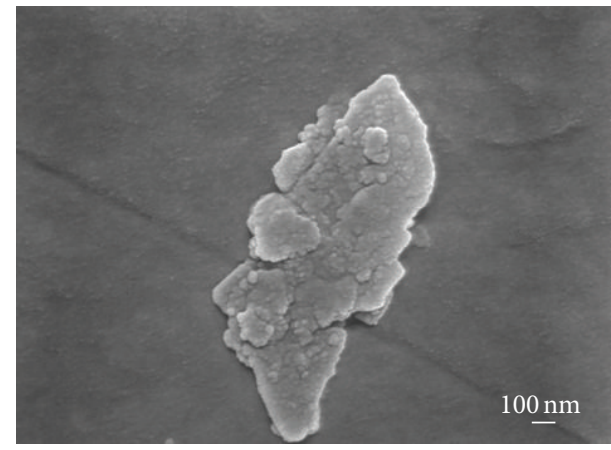

(d)

FIGURE 6: SEM images of $S$. aureus attached to (a) glass plate and (b) PLA/PU/GO (5\%) for $4 \mathrm{~h}$ incubation at $37^{\circ}$; SEM images of E. coli attached on (c) glass plate and (d) PLA/PU/GO (5\%) for $4 \mathrm{~h}$ incubation at $37^{\circ} \mathrm{C}$.

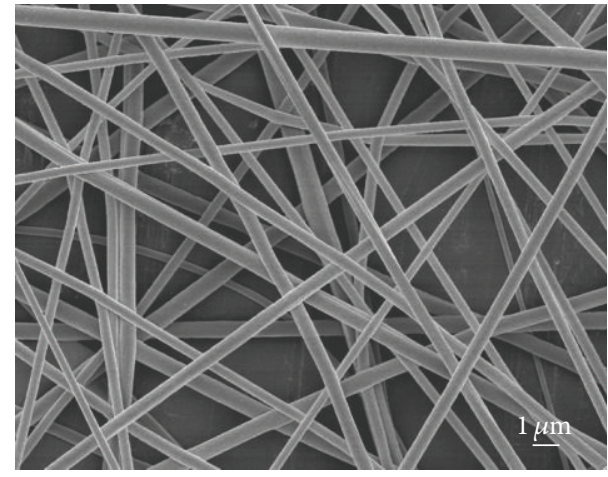

(a)

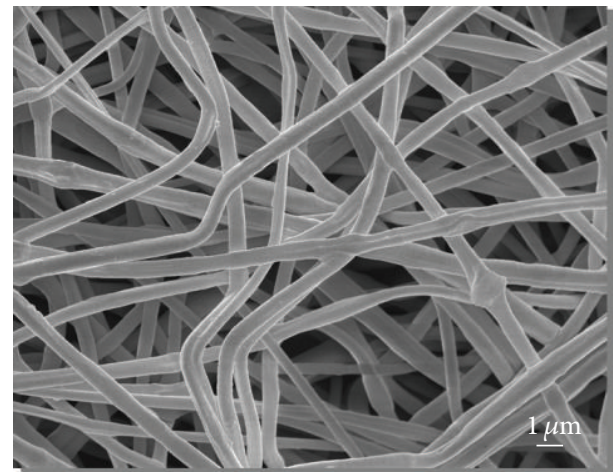

(b)

FiguRE 7: SEM images of (a) electrospun PLA/PU nanofibers and (b) electrospun PLA/PU/GO (5\%) nanofibers.

they are mixed by ultrasonication and high speed stirring, PU particles and GO sheets will preferentially stick together due to the strong interactions, as a result that $\mathrm{PU}$ particles are wrapped by GO sheets.

Figure 3 shows the XRD patterns of PLA/PU and PLA/PU/GO (5\%) films as well as the XRD pattern of powdery GO. The XRD pattern of the GO powders exhibits a characteristic diffraction peak at $11.6^{\circ}$ (the shoulder centered at $20^{\circ}$ is attributed to the glass plate, which is used as the substrate of supporting powdery sample for XRD measurement). The XRD patterns obtained from the PLA/PU/GO (5\%) film is similar to those of the PLA/PU film apart from the existence of a weak peak ascribed to the GO phase. Therefore, the XRD result further confirms the successful addition of GO sheets into PLA/PU film, which is consistent with the result coming from SEM investigations. The weakening of GO peak is due to two aspects: one is the relatively low content of GO and the other is the good dispersion of GO within the PLA/PU matrix.

Figure 4 exhibits considerable antibacterial activities when the bacteria are attached to PLA/PU/GO (3\% GO and $5 \%$ GO) for $24 \mathrm{~h}$. The incorporation of $5 \%$ GO into PLA/PU composite reduced E. coli and S. aureus growth up to $100 \%$. Moreover, the incorporation of 3\% GO reduced $E$. 


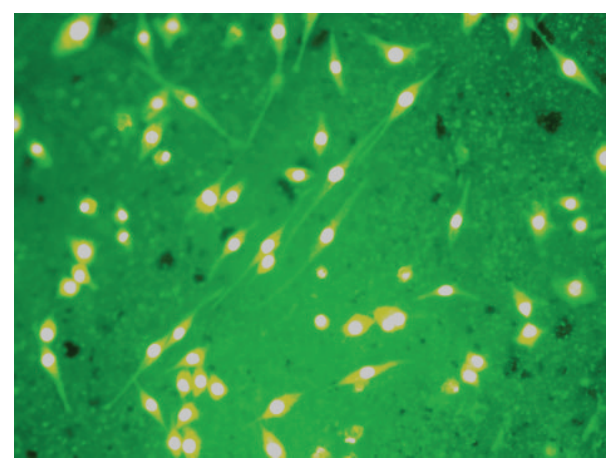

FIGURE 8: Fluorescence microscopy image of MC3T3-E1 cells grown on the electrospun PLA/PU/GO (5\%) nanofibers for $48 \mathrm{~h}$ at $37^{\circ} \mathrm{C}$. The magnification is $100 \mathrm{x}$.

coli growth up to $100 \%$ and reduces $S$. aureus growth up to $99 \%$, at $24 \mathrm{~h}$. As the control group, PLA/PU composite showed the ability to inhibit bacterial growth, but not more effective than experimental groups. Such a release profile is consistent with SEM studies in Figure 4. The introduction of GO nanoparticles may reduce the probability that the bacteria will colonize on implant surface [23].

After $4 \mathrm{~h}$ incubation with PLA/PU/GO nanocomposites of ( $3 \% \mathrm{GO}, 5 \% \mathrm{GO})$ at $37^{\circ} \mathrm{C}$, the antibacterial activity for $E$. coli decreased to $54 \%$ and $91 \%$ (Figure 5(a)), suggesting the strong inhibition ability of PLA/PU/GO nanocomposites to E. coli. We also employed a classic-colony counting method to measure the microbial viability of $S$. aureus for $4 \mathrm{~h}$. We found that viability significantly lose up to $54 \%$ and $89 \%$, respectively (Figure 5(b)). These results further confirmed that GO contents were responsible for the observed strong antibacterial effect.

Figure 6 shows that the morphology of $S$. aureus and $E$. coli when seeded on glass plates and PLA/PU/GO nanocomposites for $4 \mathrm{~h}$ at $37^{\circ} \mathrm{C}$. The control group (seeded on glass plates) displays a normal bacterial appearance of $S$. aureus and E. coli (Figures 6(a) and 6(c)). The SEM studies (Figures 6(b) and 6(d)) reveal that $S$. aureus and E. coli largely lost their original appearance; such irreversible changes might arise from the effects of either oxidative stress or physical disruption that have been related to carbon nanomaterial $[24,28]$.

Figure 7 shows the SEM micrographs of the PLA/PU and PLA/PU/GO nanofibers. It is clearly seen that ultrafine fibers have been successfully fabricated via electrospinning and all the fibers present loose three-dimensional (3D) frameworks. Also, the integration of 3D frameworks that mimic the natural extra-cellular matrix (ECM) structure is beneficial for cell adhesion and growth [30].

Figure 8 shows the fluorescence microscopy result after the cells were seeded directly on PLA/PU/GO fibrous scaffolds and incubated for $24 \mathrm{~h}$. The PLA/PU/GO nanocomposite appeared to enhance the cell adhesion and proliferation. It can be definitely attributed to the excellent intrinsic biocompatibility and the hydrophilic nature of GO materials, which can greatly promote the cell-material interaction. The soluble and nonsoluble degradation products were found to be nontoxic to bovine AF cells grown in vitro.

\section{Conclusion}

PLA/PU/GO nanocomposites passess strong antimicrobial activities and exhibit excellent biocompatibility, suggesting that such nanocomposite could be a good candidate material applied in tissue engineering territory, while simultaneously reducing the threat of bacterial colonization and subsequent chronic infection of surrounding tissue.

\section{Acknowledgments}

This paper was supported by the Open Fund of Key Laboratory of Mechanics on Disaster and Environment in Western China (201203) and the Natural Science Foundation of Gansu Province (1208RJYA036).

\section{References}

[1] R. Langer and J. P. Vacanti, “Tissue engineering," Science, vol. 260, no. 5110, pp. 920-926, 1993.

[2] V. Maquet, A. R. Boccaccini, L. Pravata, I. Notingher, and R. Jérôme, "Porous poly $(\alpha$-hydroxyacid $) /$ Bioglass composite scaffolds for bone tissue engineering," Biomaterials, vol. 25, no. 18, pp. 4185-4194, 2004.

[3] F. Yang, R. Murugan, S. Ramakrishna, X. Wang, Y.-X. Ma, and S. Wang, "Fabrication of nano-structured porous PLLA scaffold intended for nerve tissue engineering," Biomaterials, vol. 25, no. 10, pp. 1891-1900, 2004.

[4] A. R. Boccaccini, X. Chatzistavrou, J. J. Blaker, and S. N. Nazhat, "Degradable and bioactive synthetic composite scaffolds for bone tissue engineering," in Degradation of Implant Materials, pp. 111-137, Springer, New York, NY, USA, 2012.

[5] S. Y. Ivanov, R. F. Mukhametshin, A. A. Muraev, and D. V. Solodkaya, "Synthetic materials used for the substitution of bone defects," Annals of Oral \& Maxillofacial Surgery, vol. 1, no. 1, pp. $1-4,2013$.

[6] A. Aravamudhan, D. M. Ramos, J. Nip et al., "Cellulose and collagen derived micro-nano structured scaffolds for bone tissue engineering," Journal of Biomedical Nanotechnology, vol. 9, no. 4, pp. 719-731, 2013.

[7] C. Liu, Z. Xia, and J. T. Czernuszka, "Design and development of three-dimensional scaffolds for tissue engineering," Chemical Engineering Research and Design, vol. 85, no. 7 A, pp. 1051-1064, 2007.

[8] N. Kébir, I. Campistron, A. Laguerre, J.-F. Pilard, C. Bunel, and T. Jouenne, "Use of telechelic cis-1,4-polyisoprene cationomers in the synthesis of antibacterial ionic polyurethanes and copolyurethanes bearing ammonium groups," Biomaterials, vol. 28, no. 29, pp. 4200-4208, 2007.

[9] G. N. Belibasakis, T. Thurnheer, and N. Bostanci, "Infections associated with implanted dental devices," in Biomaterials Associated Infection, pp. 249-271, Springer, New York, NY, USA, 2013.

[10] A. G. Gristina, "Biomaterial-centered infection: microbial adhesion versus tissue integration," Science, vol. 237, no. 4822, pp. 1588-1595, 1987. 
[11] P. M. Sivakumar, S. Cometa, M. Alderighi, V. Prabhawathi, M. Doble, and F. Chiellini, "Chalcone embedded polyurethanes as a biomaterial: synthesis, characterization and antibacterial adhesion," Carbohydrate Polymers, vol. 87, no. 1, pp. 353-360, 2012.

[12] K. Feng, H. Sun, M. A. Bradley, E. J. Dupler, W. V. Giannobile, and P. X. Ma, "Novel antibacterial nanofibrous PLLA scaffolds," Journal of Controlled Release, vol. 146, no. 3, pp. 363-369, 2010.

[13] Z.-C. Xing, W.-P. Chae, J.-Y. Baek, M.-J. Choi, Y. Jung, and I.$\mathrm{K}$. Kang, "In vitro assessment of antibacterial activity and cytocompatibility of silver-containing PHBV nanofibrous scaffolds for tissue engineering," Biomacromolecules, vol. 11, no. 5, pp. 1248-1253, 2010.

[14] J. B. Lee, H. N. Park, W. K. Ko et al., "Poly(L-lactic acid)/hydroxyapatite nanocylinders as nanofibrous structure for bone tissue engineering scaffolds," Journal of Biomedical Nanotechnology, vol. 9, no. 3, pp. 424-429, 2013.

[15] M. Yeganegi, R. A. Kandel, and J. P. Santerre, "Characterization of a biodegradable electrospun polyurethane nanofiber scaffold: mechanical properties and cytotoxicity," Acta Biomaterialia, vol. 6, no. 10, pp. 3847-3855, 2010.

[16] H. Bakhshi, H. Yeganeh, and S. Mehdipour-Ataei, "Synthesis and evaluation of antibacterial polyurethane coatings made from soybean oil functionalized with dimethylphenylammonium iodide and hydroxyl groups," Journal of Biomedical Materials Research A, vol. 101, no. 6, pp. 1599-1611, 2013.

[17] N. Fong, A. Simmons, and L. A. Poole-Warren, "Antibacterial polyurethane nanocomposites using chlorhexidine diacetate as an organic modifier," Acta Biomaterialia, vol. 6, no. 7, pp. 25542561, 2010.

[18] A. S. Rowlands, S. A. Lim, D. Martin, and J. J. Cooper-White, "Polyurethane/poly(lactic-co-glycolic) acid composite scaffolds fabricated by thermally induced phase separation," Biomaterials, vol. 28, no. 12, pp. 2109-2121, 2007.

[19] K. S. Novoselov, A. K. Geim, S. V. Morozov et al., "Electric field in atomically thin carbon films," Science, vol. 306, no. 5696, pp. 666-669, 2004.

[20] M. Song, L. Yu, and Y. Wu, "Simple synthesis and enhanced performance of graphene oxide-gold composites," Journal of Nanomaterials, vol. 2012, Article ID 135138, 5 pages, 2012.

[21] J. Chen, G. Zhang, B. Luo, D. Sun, X. Yan, and Q. Xue, "Surface amorphization and deoxygenation of graphene oxide paper by Ti ion implantation," Carbon, vol. 49, no. 9, pp. 3141-3147, 2011.

[22] Y. Zhu, S. Murali, W. Cai et al., "Graphene and graphene oxide: synthesis, properties, and applications," Advanced Materials, vol. 22, no. 35, pp. 3906-3924, 2010.

[23] J.-W. Jiang, J.-S. Wang, and B. Li, "Young's modulus of graphene: a molecular dynamics study," Physical Review B, vol. 80, no. 11, Article ID 113405, 2009.

[24] Y. Shi, W. Fang, K. Zhang, W. Zhang, and L.-J. Li, "Photoelectrical response in single-layer graphene transistors," Small, vol. 5, no. 17, pp. 2005-2011, 2009.

[25] X. Yan, J. Chen, J. Yang, Q. Xue, and P. Miele, "Fabrication of free-standing, electrochemically active, and biocompatible graphene oxide-polyaniline and graphene-polyaniline hybrid papers," ACS Applied Materials and Interfaces, vol. 2, no. 9, pp. 2521-2529, 2010.

[26] Y. Xu, W. Hong, H. Bai, C. Li, and G. Shi, "Strong and ductile poly(vinyl alcohol)/graphene oxide composite films with a layered structure," Carbon, vol. 47, no. 15, pp. 3538-3543, 2009.
[27] Y. Feng, X. Zhang, Y. Shen, K. Yoshino, and W. Feng, "A mechanically strong, flexible and conductive film based on bacterial cellulose/graphene nanocomposite," Carbohydrate Polymers, vol. 87, no. 1, pp. 644-649, 2012.

[28] L. M. Veca, F. Lu, M. J. Meziani et al., "Polymer functionalization and solubilization of carbon nanosheets," Chemical Communications, no. 18, pp. 2565-2567, 2009.

[29] H. J. Salavagione, M. A. Gómez, and G. Martínez, "Polymeric modification of graphene through esterification of graphite oxide and poly(vinyl alcohol)," Macromolecules, vol. 42, no. 17, pp. 6331-6334, 2009.

[30] Y. Chen, Y. Qi, Z. Tai, X. Yan, F. Zhu, and Q. Xue, "Preparation, mechanical properties and biocompatibility of graphene oxide/ultrahigh molecular weight polyethylene composites," European Polymer Journal, vol. 48, no. 6, pp. 1026-1033, 2012.

[31] Y. Xu, H. Bai, G. Lu, C. Li, and G. Shi, "Flexible graphene films via the filtration of water-soluble noncovalent functionalized graphene sheets," Journal of the American Chemical Society, vol. 130, no. 18, pp. 5856-5857, 2008.

[32] W. Hu, C. Peng, W. Luo et al., "Graphene-based antibacterial paper," ACS Nano, vol. 4, no. 7, pp. 4317-4323, 2010.

[33] O. Akhavan and E. Ghaderi, "Toxicity of graphene and graphene oxide nanowalls against bacteria," ACS Nano, vol. 4, no. 10, pp. 5731-5736, 2010.

[34] J. Shen, M. Shi, N. Li et al., "Facile synthesis and application of Ag-chemically converted graphene nanocomposite," Nano Research, vol. 3, no. 5, pp. 339-349, 2010.

[35] D. Sarkar, J.-C. Yang, A. Sen Gupta, and S. T. Lopina, "Synthesis and characterization of L-tyrosine based polyurethanes for biomaterial applications," Journal of Biomedical Materials Research A, vol. 90, no. 1, pp. 263-271, 2009.

[36] Y. Zhang, C. Wang, X. Pei, Q. Wang, and T. Wang, "Shape memory polyurethanes containing azo exhibiting photoisomerization function," Journal of Materials Chemistry, vol. 20, no. 44, pp. 9976-9981, 2010.

[37] W. S. Hummers Jr. and R. E. Offeman, "Preparation of graphitic oxide," Journal of the American Chemical Society, vol. 80, no. 6, p. 1339, 1958.

[38] Y. Xu, H. Bai, G. Lu, C. Li, and G. Shi, "Flexible graphene films via the filtration of water-soluble noncovalent functionalized graphene sheets," Journal of the American Chemical Society, vol. 130, no. 18, pp. 5856-5857, 2008.

[39] D. R. Dreyer, S. Park, C. W. Bielawski, and R. S. Ruoff, "The chemistry of graphene oxide," Chemical Society reviews, vol. 39, no. 1, pp. 228-240, 2010.

[40] X. Zhou, Y. Zhang, C. Wang et al., "Photo-fenton reaction of graphene oxide: a new strategy to prepare graphene quantum dots for dna cleavage," ACS Nano, vol. 6, no. 8, pp. 6592-6599, 2012.

[41] A. K. Geim, “Graphene: status and prospects," Science, vol. 324, no. 5934, pp. 1530-1534, 2009.

[42] D. Cai and M. Song, "Preparation of fully exfoliated graphite oxide nanoplatelets in organic solvents," Journal of Materials Chemistry, vol. 17, no. 35, pp. 3678-3680, 2007. 

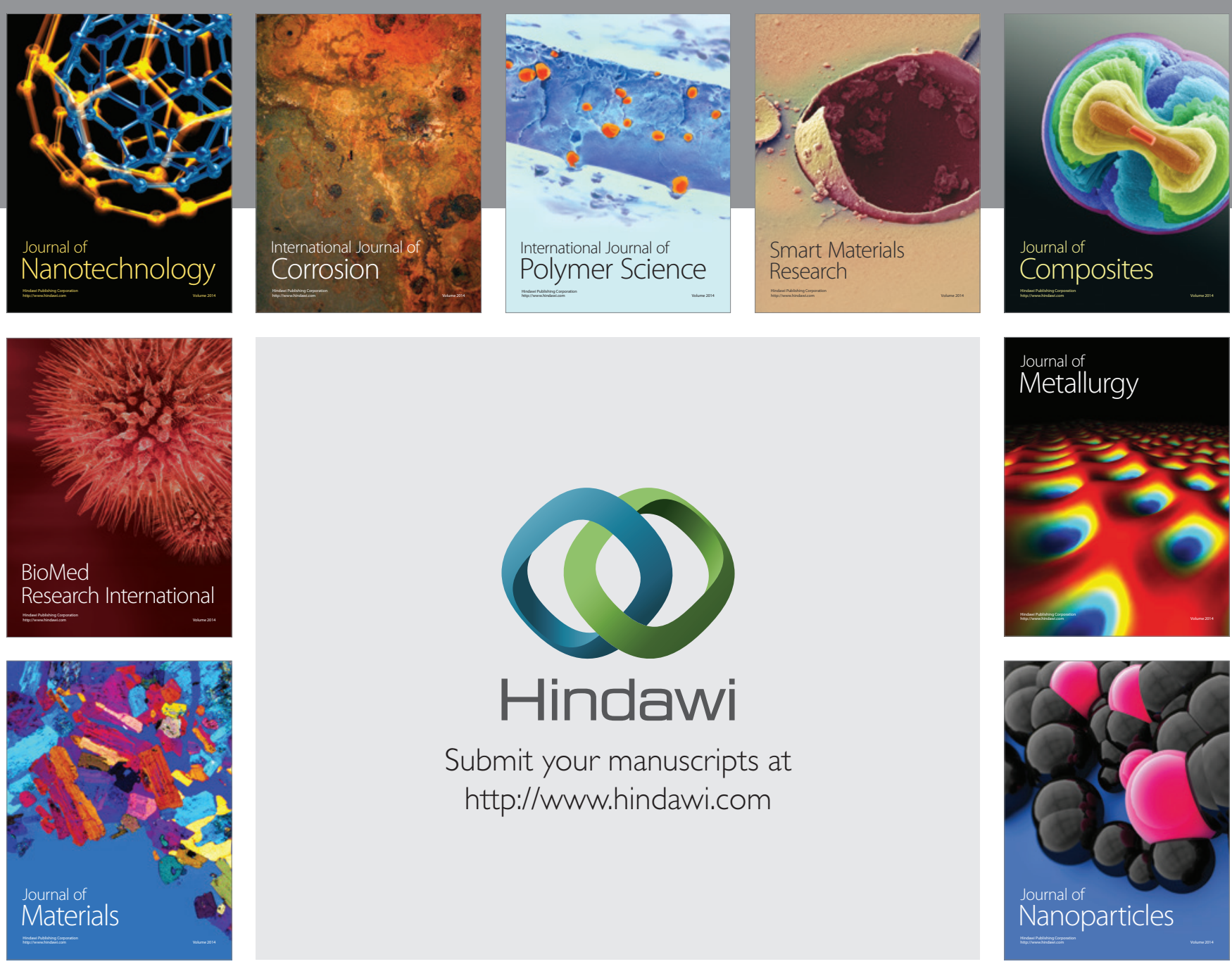

Submit your manuscripts at http://www.hindawi.com
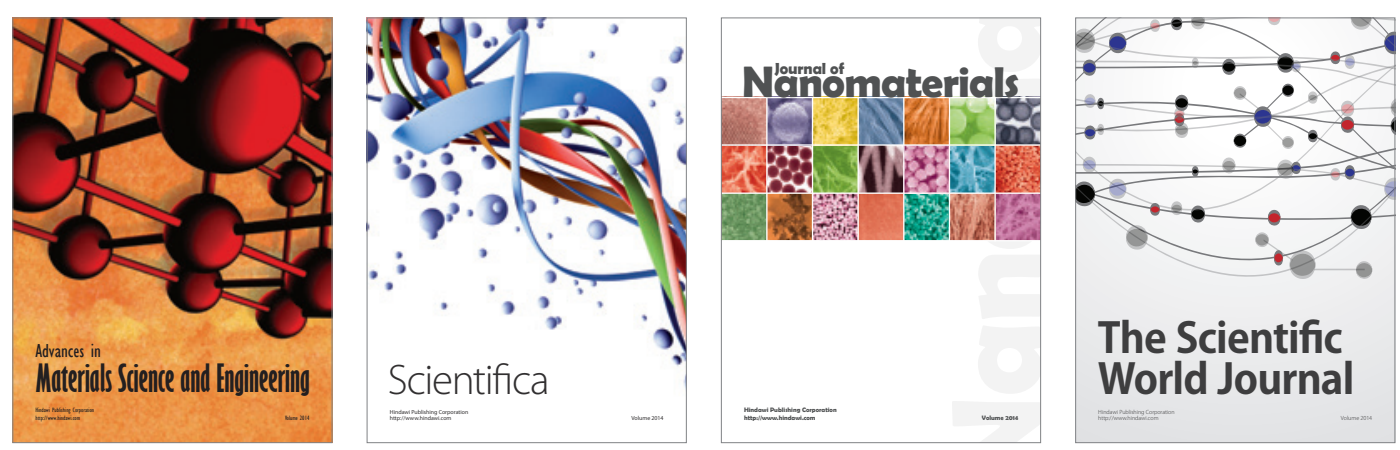

\section{The Scientific World Journal}
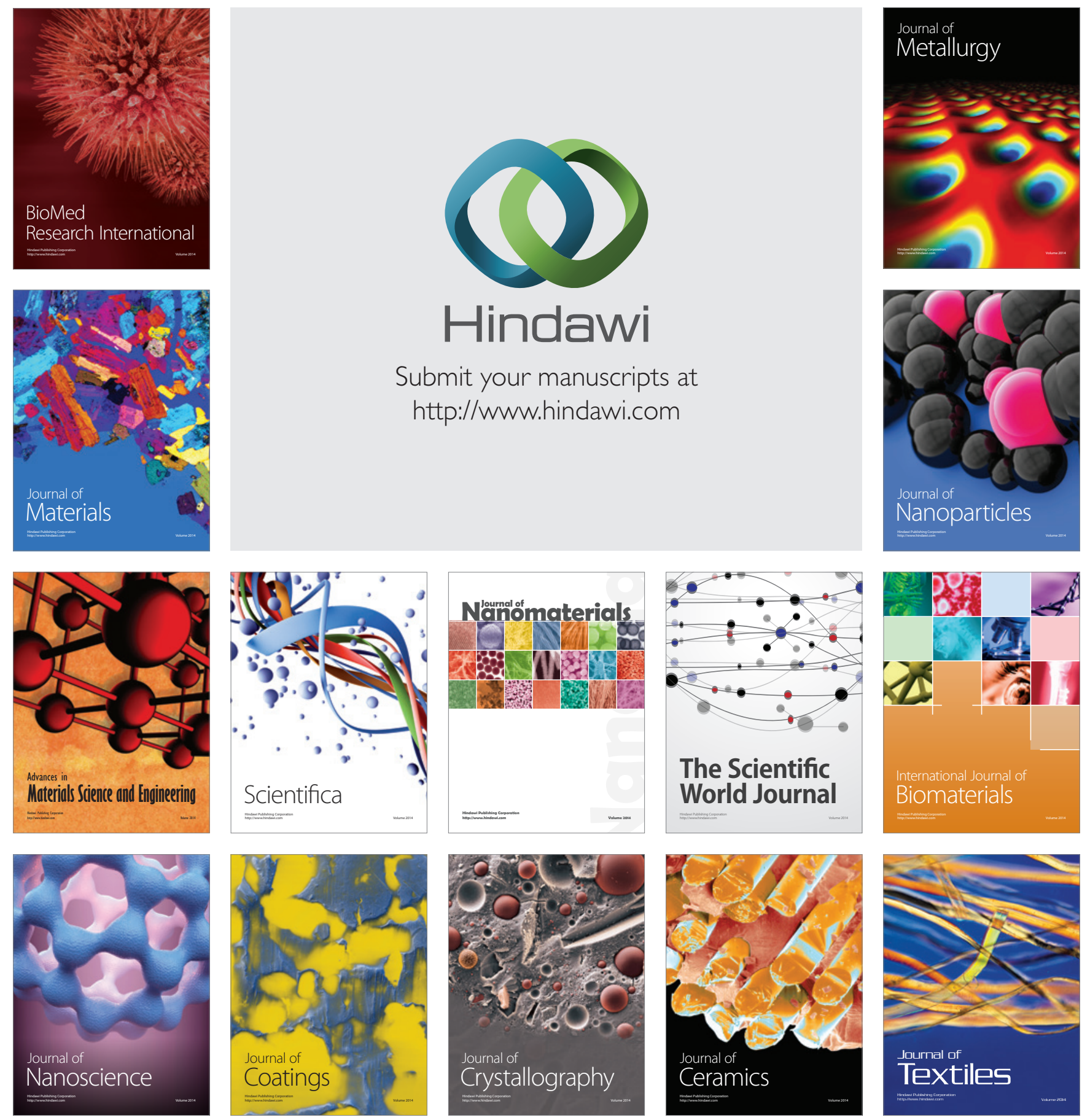